Isaac Cesar S. De Guzman, MD

Department of Otorhinolaryngology Head and Neck Surgery

De La Salle University Medical Center
Correspondence: Dr. Isaac Cesar S. De Guzman

Department of Otorhinolaryngology

Head and Neck Surgery

De La Salle University Medical Center

Congressional Avenue, Dasmarinas City, Cavite 4114

Philippines

Phone: (046) 416-0226 local 1340

Email: ayceedg@gmail.com

The author declared that this represents original material that is not being considered for publication or has not been published or accepted for publication elsewhere in full or in part in in print or electronic media; that the manuscript has been read and approved by the authors, that the requirements for authorship have been met by the author, and that the author believes that the manuscript represents honest work.

Disclosures: The author signed a disclosure that there are no financial or other (including personal) relationships, intellectual, passion, political or religious beliefs, and institutional affiliations that might lead to a conflict of interest.

Presented at the Philippine Society of Otolaryngology-Head and Neck Surgery, Analytical Research Contest, November 9, 2017, Menarini Office, Bonifacio High Street, Taguig City.

\section{Tracheal Diameter Estimates Using Age-Related Formula Versus Radiographic Findings: Which Approximates the Actual Tracheostomy Tube in Pediatric Patients?}

\begin{abstract}
Objective: To compare actual tracheostomy tube sizes with estimated endotracheal tube sizes using age-related formula and tracheal diameter from preoperative radiographs among pediatric Filipino patients aged 0-18 years old undergoing tracheostomy.
\end{abstract}

\section{Methods:}

$\begin{array}{ll}\text { Design: } & \text { Review of records } \\ \text { Setting: } & \text { Tertiary Private University Hospital in Dasmarinas, Cavite, Philippines } \\ \text { Patients: } & \text { Pediatric patients regardless of gender, aged } 0 \text { to } 18 \text { years old with }\end{array}$
a preoperative radiograph of the tracheal and who subsequently underwent tracheostomy anytime from January 1, 2007 to December 31, 2016 were considered for inclusion. Radiographs were measured, endotracheal tube sizes were computed using age-related formula and recorded tracheotomy tube sizes were retrieved.

Results: Twenty-two patients (12 males, 10 females) aged 10 months to 18-years-old (median age: 11 years) were included in the study. Mean tube sizes were $6.46 \mathrm{~mm}$ (+/- $1.492 \mathrm{SD}$ ) for agerelated formula, $5.67 \mathrm{~mm}$ (+/- $1.1849 \mathrm{SD})$ for radiograph-based estimation and 5.0 for actual tracheostomy tube inserted in each patient. The Bland-Altman plot showed the bias estimate at 0.7913 and the lower and upper limits of agreement at -1.3598 and 2.9423 (confidence level 95\% or 2 standard deviations away from the mean).

Conclusion: The average value derived from radiograph-based estimation is less than the corresponding average value from age-related formula. There is a significant difference between age-related formula-based estimation and actual tracheostomy tube inserted. Since the range of differences between the two estimation methods is high, these results imply that the bias or the difference between measures from the two methods is not consistent with the two methods exhibiting very poor agreement.

Keywords: Tracheostomy, Intubation, Intra Tracheal, Penlington Formula, Trachea Radiograph Measurement, age related formula for endotracheal tube estimation 
Tracheostomy is a commonly performed surgical procedure to manage the airway. Choosing the correctly sized tracheostomy tube is vital since failing to predict a suitable tube size may cause potentially devastating complications. This concern is even more critical in children due to the smaller caliber of the pediatric airway and the potentially lifelong impact of airway injury.

There are very limited studies published on techniques used for estimation of tracheostomy tube size and methods for matching airway tube size to tracheal diameter are limited. ${ }^{1}$ Anesthesiologists sometimes use the size of the fifth digit. ${ }^{2}$ Others use several formulas, the most common being $(16+$ age $) / 4$ or variations thereof ${ }^{2}$ to predict the internal diameter of the tube and the corresponding tube size. This age-based formula is not always reliable because of differences in children's physical development. ${ }^{3}$ Park et al. used an airway radiographbased formula to predict the appropriate endotracheal tube size in children aged 3 to 6 years old, measuring the diameter of the tracheal airway column using a routine chest AP radiograph. ${ }^{4}$ To the best of our knowledge based on a search of PubMed (MEDLINE), HERDIN and Google Scholar, there is no published study to date that compares the results obtained from the age-related formula with those from airway radiographs in terms of selecting the correct tracheostomy tube size.

Thus, the aim of this study is to compare actual tracheostomy tube sizes with estimated endotracheal tube sizes using age-related formula and tracheal diameter from preoperative radiographs among pediatric Filipino patients undergoing tracheostomy.

\section{Study Design}

\section{METHODS}

With approval of the Institutional Independent Ethics Committee, this study reviewed patient records at the De La Salle University Medical Center in collaboration with the Departments of Ear, Nose, Throat Head and Neck Surgery (ENT-HNS), Anesthesia, Radiology, and Medical Records.

\section{Participants}

Using the WHO definition of children, pediatric patients regardless of gender, aged 0 to 18 years old who underwent tracheostomy between January 1, 2007 and December 31, 2016 and had a preoperative radiograph of the trachea were considered for inclusion in the study. Exclusion criteria included conditions which may affect normal tracheal size like tracheal tumors, mediastinal tumors, congenital airway anomalies and those with poor quality radiographs.

A list of all pediatric patients who underwent tracheostomy was obtained from the Operating Room Complex Records. The availability of operative notes and chest $X$ ray results in the medical records of these patients were then ascertained by chart review starting in December 2016 and sequentially going back in time. A sample size of 22 patients was calculated to detect an effect size of 0.65 with a $95 \%$ confidence level and power of $80 \%$. Standard deviation of the difference was set at $+/-1 \cdot 0 .{ }^{3}$ Patients were included until the required sample size was reached. Preoperative radiographs were then retrieved from the hospital radiology picture archive system. The informed consents signed by the parents cover use of patient information for research.

\section{Data Collection Procedure}

A data abstraction form was developed based on study objectives. Information was abstracted from the medical charts. Age based estimation of tracheostomy tube size used the Penlington formula for estimating endotracheal tube size wherein internal diameter, ID $=(16+$ age $) / 4$. Radiographic estimates of the tracheal diameter of the subjects was estimated by a single radiology resident using the preoperative chest radiograph by measuring the transverse width of the trachea between $3^{\text {rd }}$ and $4^{\text {th }}$ tracheal ring in millimeters on the AP hospital radiology picture archive system. This measurement corresponds to the outer diameter of the tracheostomy tube. The radiologist was blinded to the records of the actual tube size and the results of the Penlington formula.

\section{Data Analysis}

Data was encoded in Microsoft Excel v. 16.15 (180709) (Microsoft Corp., Redmond, WA, USA) and analyzed using SAS for Windows v 6.0 (SAS Instiute Inc., Cary, North Carolina, USA). Measures of central tendency and dispersion were used to summarize continuous variables including age, weight, height and tracheostomy tube measurements including age-related estimation, preoperative radiograph and actual size of tracheostomy tube used. Frequency tables and percentage distributions were used to summarize age group and gender of subjects. Shapiro-Wilk test was used to determine normality of data. Wilcoxon-signed rank test was used to test any significant difference in tracheostomy tube size between age-related formula and methods of Penlington formula results. The mean difference of the 2 methods of estimation was computed with a $95 \%$ confidence interval. A BlandAltman plot was used to describe the differences between internal diameters computed using the Penlington formula and internal diameters corresponding to measurements obtained from the chest radiographs. A p-value less than .05 was considered statistically significant. 


\section{ORIGINAL ARTICLES}

\section{RESULTS}

In total, 23 patients were considered upon reviewing records from January 2007 to December 2016; one patient was excluded because of unavailable $x$-ray image. Table 1 below shows that among the 22 patients, 12 were males and 10 females. Ages ranged from 10 months to 18 years (median 11 years).

Table 1. Frequency Distributions and Summary Statistics

\begin{tabular}{|l|r|r|r|r|r|}
\multicolumn{1}{|c|}{ Variables } & Mean & Std. Dev. & Median & Min & Max \\
\hline Age (yrs.) & 9.86 & 5.97 & 11 & 0 & 18 \\
\hline Weight (kg) & 30.21 & 16.57 & 30 & 8.0 & 60 \\
\hline $\begin{array}{l}\text { Age-related formula } \\
\text { estimate (mm) }\end{array}$ & 6.46 & 1.49 & 6.75 & 4.21 & 8.5 \\
\hline $\begin{array}{l}\text { Radiography-based } \\
\text { estimate (mm) }\end{array}$ & 5.67 & 1.19 & 5.25 & 3.5 & 8.9 \\
\hline $\begin{array}{l}\text { Actual TT tube size } \\
\text { (mm) }\end{array}$ & 5.01 & 1.21 & 5.0 & 3.0 & 6.4 \\
\hline
\end{tabular}

As observed, the diameters of tracheostomy tubes used among these patients ranged from $3 \mathrm{~mm}$ to $6.4 \mathrm{~mm}$ with an average diameter of 5.01 (SD, 1.21). The average actual tracheostomy tube sizes were less than the average estimates derived from the two methods. Also, the minimum and the maximum values from actual tracheostomy tube sizes were less than the minimum and maximum estimates being observed from the two methods of estimation.

Estimates derived from the age-related formula ranged from 4.20 $\mathrm{mm}$ to $8.50 \mathrm{~mm}$ with an average diameter of $6.46 \mathrm{~mm}$ (SD, 1.49). On the other hand, estimates based on radiography ranged from $3.5 \mathrm{~mm}$ to 8.9 $\mathrm{mm}$ with an average diameter of $5.67 \mathrm{~mm}$ (SD, 1.18). Table 2 shows that there were indeed significant differences between estimates as well as between each estimate and tracheotomy tube size actually used.

Table 2. Comparing Means between Age-related formula estimates, radiography-based estimates and Actual Tracheostomy Tube Sizes

\begin{tabular}{|l|c|c|}
\hline \multicolumn{1}{|c|}{ Wilcoxon Signed-rank test } & Test Statistic & P-value \\
\hline $\begin{array}{l}\text { Age-related formula estimates } \\
\text { vs radiograph-based estimates }\end{array}$ & 2.763 & .0057 \\
\hline $\begin{array}{l}\text { Actual tracheostomy tube sizes vs } \\
\text { age-related formula estimates }\end{array}$ & 4.015 & .0001 \\
\hline $\begin{array}{l}\text { Actual tracheostomy tube sizes vs } \\
\text { d radiography-based estimates }\end{array}$ & -2.446 & .0143 \\
\hline
\end{tabular}

Pearson's Tests for Correlation yielded a coefficient of correlation of 0.838 (P-value $<.0001$ ) and a coefficient of determinations $\left(R^{2}\right)$ of $70.29 \%$ between age-related formula estimates and actual tracheostomy tube sizes. In contrast, Pearson's test on radiograph-based estimation vs. actual tracheostomy tube sizes yielded a coefficient of correlation of 0.511 (P-value <.0151, R2 26.10\%). Figure 1 which correlates the two estimates shows that radiography-based estimates are significantly lower than the corresponding age-related formula-based estimates ( $p$ $=.0028$ ).

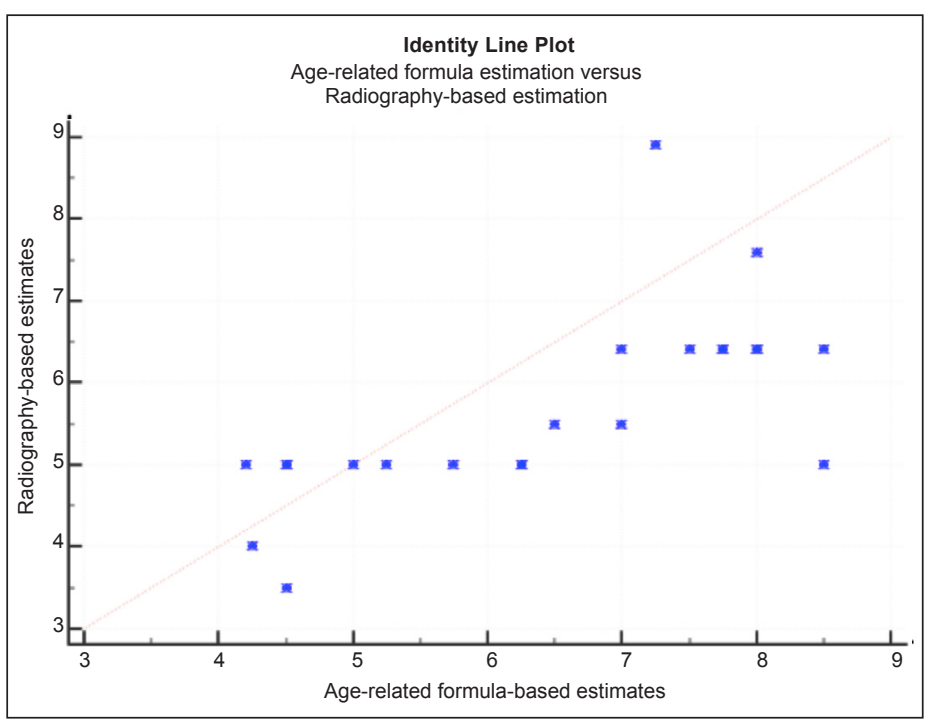

Figure 1. Identity line plot for age related formula versus radiograph-based estimation. Note numerous data points below the identity line implying that radiograph-based estimation exhibits a significant negative bias compared to age-related formula-based estimation $(p=.0028)$

Figure 2 shows that the differences between age-based and radiograph-based estimates increased as the means of these two estimates increased. This supports the previous finding that tracheotomy tube size was more strongly correlated with age-based

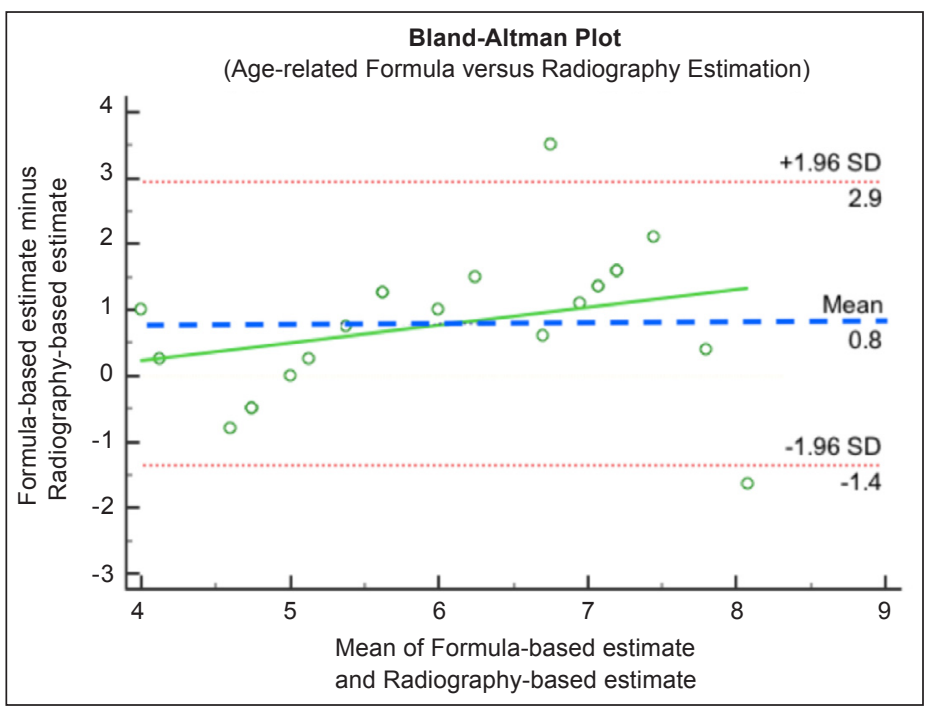

Figure 2. Bland-Altman plot for age-related formula versus radiograph-based estimation Solid green line, trend line of data points; blue dashed line, mean difference; red dotted lines, standard deviation or limits of agreement; green circles, data points of patients. 
estimates than with radiograph-based estimates and that radiographbased estimates are lower than age - based estimates. Note that the bias estimate is 0.7913 , with a $95 \%$ confidence interval of 0.3046 to 1.2779 .

\section{DISCUSSION}

Our study found that there is a significant difference between age-related formula-based estimation and actual tracheostomy tube inserted, as well as between age-related formula and radiographbased estimation. The average value derived from radiograph-based estimation is less than the corresponding average value from agerelated formula. This difference is also clinically important since the estimates differ one tracheostomy tube size bigger than the actual tube size used.

The difference in the means of the radiograph-based formula and age-related formula may be due to the small sample size used in this study, making variances in patient characteristics more significant. The difference in the mean of the size estimates for the age-related formula and radiograph-based estimation $(0.79 \mathrm{~mm})$ is statistically significant and clinically important as well since the increment of internal diameters between sizes of tracheostomy tubes is only $0.5 \mathrm{~mm}$. The results support the findings of Park et al. comparing radiographbased formula and age-related formula in estimating endotracheal tube size. The study involved a larger sample size and observed that there is a smaller difference between the actual tube size inserted and radiograph-based estimation than the difference between actual tube size inserted and age-related formula-based estimation. ${ }^{4}$

The correlation analysis indicates there are indeed significant differences between the measurements derived from age-related formula estimation and radiograph-based estimation. The agerelated formula estimates exhibit stronger correlation with the actual tracheostomy tube sizes. Also, age-related formula estimation provides higher predictive capability against radiograph-based estimation in explaining the variability present among actual tracheostomy tube sizes. These findings are expected since the age-related formula estimation method is already being practiced in our institution compared to the proposed estimation method based on radiography.

However, it cannot be concluded yet at this stage whether radiograph-based estimation can be used as an alternative estimation procedure in predicting actual tracheostomy tube sizes. The sizes may differ significantly but the difference can be considered important if it is constant and has a pattern.

The identity line plot allows us to quickly see if there is a fixed bias or not by directly comparing the radiograph-based estimation to the age-related formula-based estimation. Since the data points are widely scattered away from the identity line, this makes the radiographbased estimation not a relevant substitute for the age-based formula. Almost all of the data points lie below the identity line. This implies that radiograph-based estimation exhibits a negative bias compared to the age-related formula-based estimation. That is, it is expected that radiograph-based estimation will constantly yield smaller estimates compared to age-related formula estimation. Adding a constant value could probably address this bias that will then make radiograph measurements a possible alternative to the age-based formula. Further research especially with a larger sample size may allow the formulation of an equation for computing the proper tracheostomy tube size using the tracheal diameter measured from the radiograph.

As observed in the Bland Altman Analysis, the points are widely scattered within the limits of agreement and unfortunately, there are two points lying outside the limits of agreement. Since the range of differences between the radiograph-based and age-related formula methods is rather high, these results imply that the bias or the difference between measures from the two methods is not consistent. These results suggest that radiograph-based estimation exhibits very poor agreement with age-related formula estimation suggesting that they cannot be used interchangeably.

There are no standard formulas for determining proper tracheostomy or endotracheal tube size. Due to limitations, chest $X$-ray images are frequently used to measure the transverse tracheal width. The measurement of transverse tracheal width is usually performed using non-invasive methods such as chest $\mathrm{X}$-ray, $\mathrm{CT}^{5}$ $\mathrm{MRI}^{6}$, or ultrasonography. ${ }^{7}$ However, high-quality laryngeal images provided by $\mathrm{CT}$ and MRI are not routinely obtained and the quality of ultrasonography is operator dependent. ${ }^{6}$ Furthermore, in adults, neither height nor weight predicts transverse tracheal width.,9 Different algorithms and formulae have been proposed to choose the best-fitting size of the tracheal tube. The most widely accepted is the age-based formula wherein the inner diameter in $\mathrm{mm}=(16+$ age $) / 4$. This calculation overestimates the correct size in more than one in four cases. ${ }^{10}$

The age-based formula is reliable and easily applied and accepted for routine anesthesia in the pediatric population. ${ }^{11}$ However, the age-related formula considers only the patients age with no regard for the actual size of the patient. On the other hand, the radiographbased formula measures the trachea of the patient directly. Smaller tracheostomy tubes inserted can also be attributed to the physician's personal choice such as surgical convenience. Also, inserting a tube too large for the lumen may compromise mucosal vascular supply resulting in ischemia and necrosis along the lining of the trachea. 
The major limitation of this study is the lack of available patients in our institution caused by the low number of pediatric patients undergoing tracheostomy. Some patients also have the procedure performed bedside due to lack of funds (and are not listed in the operating room records). Strictly speaking, 18-year-old patients do not have a pediatric sized trachea. However, the WHO definition of pediatric patients was adapted in order to be consistent with other studies in children. In the future, a follow up study with greater sample size can be done. Results can be used to create a regression analysis and may provide a new formula for estimating tracheostomy tube size.

In conclusion, the average value derived from radiograph-based estimation is less than the corresponding average value from agerelated formula. There is a significant difference between age-related formula-based estimation and actual tracheostomy tube inserted. The tracheal size measurement obtained from radiographs is significantly different from but consistently larger than the actual tracheostomy tube size. If we were to correct for this difference, then a tube one size larger should be selected (with consideration for patient characteristics other than age). Since the range of differences between the two estimation methods is high, these results imply that the bias or the difference between measures from the two methods is not consistent with the two methods exhibiting very poor agreement.

\section{ACKNOWLEDGEMENTS}

I would like to acknowledge Dr. Jose Lito Acuin, the department research coordinator and Dr. Patrick Pardo, the department resident training officer, for providing assistance in writing this article. They served as my scientific advisor and critically reviewed the study proposal. I would also like to thank the Radiology Resident who helped with the data collection for the measurements of the radiographs, Dr. Gale Malbas. Also, I would like to acknowledge Prof. Danaida B. Marcelo for providing help with the statistical analysis of the results.

\section{REFERENCES}

1. Behl S, Watt JW. Prediction of tracheostomy tube size for paediatric long term ventilation: an audit of children with spinal cord injury. Br J Anesth. 2005 Jan; 94(1):88-91. DOl: 10.1093/bja/ aeh296; PMID: 15486000.

2. Barash PG. Clinical Anesthesia. 7th ed. Philadelphia: Lippincott Williams \& Wilkins, A Wolters Kluwer Business. 2013. Chapter 42. p. 1237. NLM ID: 101598359.

3. Park HP. Appropriate tube size selection based on radiological images. Korean J Anesthesiol. 2014 Mar; 66(3): 181-182. DOI: 10.4097/kjae.2014.66.3.181; PMID: 24729837 PMCID: PMC3983411.

4. Park HP, Hwang JW, Lee JH, Nahm FS, Park SH, Oh AY, et al. Predicting the appropriate uncuffed endotracheal tube size for children: a radiograph-based formula versus two age-based formulas. J Clin Anesth. 2013 Aug; 25(5):384-387. DOI: 10.1016/j.jclinane.2013.01.015; PMID: 23965215.

5. Chen SJ, Shih TT, Liu KL, Chiu IS, Wu MH, Chen HY, et al. Measurement of tracheal size in children with congenital heart disease by computed tomography. Ann Thorac Surg. 2004 Apr; 77(4): 1216-1221. DOI: 10.1016/j.athoracsur.2003.08.002; PMID: 15063238.

6. Lakhal K, Delplace X, Cottier JP, Tranquart F, Sauvagnac X, Mercier C, et al. The feasibility of ultrasound to assess subglottic diameter. Anesth Analg. 2007 Mar; 104(3): 611-614. DOl: 10.1213/01.ane.0000260136.53694.fe; PMID: 17312218.

7. Carp H, Bundy A. A preliminary study of the ultrasound examination of the vocal cords and larynx. Anesth Analg. 1992 Oct; 75(4): 639-640. PMID: 1530186.

8. Seymour AH, Prakash N. A cadaver study to measure the adult glottis and subglottis: defining a problem associated with the use of double-lumen tubes. J Cardiothorac Vasc Anesth. 2002 Apr; 16(2): 196-198. PMID: 11957170.

9. Randestad A, Lindholm CE, Fabian P. Dimensions of the cricoid cartilage and the trachea. Laryngoscope. 2000 Nov; 110(11): 1957-1961. DOI: 10.1097/00005537-200011000-00036; PMID: 11081618.

10. Mostafa SM. Variation in subglottic size in children. Proc R Soc Med. 1976 Nov; 69(11):793-95. PMID: 1005455 PMCID: PMC1864717.

11. Turkistani A, Abdullah KM, Delvi B, Al-Mazroua KA. The 'best fit' endotracheal tube in children comparison of four formulae. Middle East J Anaesthesiol. 2009 Oct; 20 (3):383-7. PMID: 19950731.
Koha BV, Jeon IS, Andre JM, MacKay I, Smith RM. Postintubation croup in children. Anesth Analg 1977 Jul-Aug; 56(4):501-5. PMID: 560135.

12. Lee KW, Templeton JJ, Dougal RM. Tracheal tube size and postintubation croup in children. Anesthes. 1980; 53:325.

13. Magill IW. Technique in endotracheal anaesthesia. Br Med J. 1930 Nov 15; 2(3645): 817-9. PMID 20775829 PMCID: PMC2451624.

14. Mir F, Sandhu G, Poncia J. Size matters: choosing the right tracheal tube. The Association of Anaesthetists of Great Britain and Ireland. Anaesthesia. 2012 Dec; 67(12): 1402-1403. DOI: 10.1111/anae.12026.

15. Brown ES. Resistance factors in pediatric endotracheal tubes and connectors. Anesth Analg. 1971 May-Jun; 50(3):355-60. PMID: 5103767

16. Divatia JV, Bhowmick K. Complications of endotracheal intubation and other airway management procedures. Indian J Anaesth. 2005; 49 (4): 308-318.

17. Shih MH, Chung CY, Su BC, Hung CT, Wong SY, Wong TK. Accuracy of a new body length-based formula for predicting tracheal tube size in Chinese children. Chang Gung Med J. 2008 May-Jun; 31(3): 276-80. PMID: 18782950.

18. Karsli CH, Isaac LA, Roy WL. Induction of anesthesia. In: Bissonnette B, Dalens BJ (editors). Pediatric Anaesthesia Principles and Practice (edition 1) 2002. New York, NY, McGraw-Hill. pp. 483-527.

19. Litman RS, Keon TP. Postintubation croup in children. Anesthesiology. 1991 Dec; 75(6):1122-3. PMID: 1741506.

20. Finholt DA, Henry DB, Raphaely RC. Factors affecting leak around tracheal tubes in children. Can Anaesth Soc J. 1985 Jul; 32(4): 326-329. PMID: 4027762.

21. Schwartz RE, Stayer SA, Pasquariello CA. Tracheal tube leak test-is there inter-observer agreement? Can J Anaesth, 1993 Nov; 40(11): 1049-1052. DOI: 10.1007/BF03009476; PMID: 8269566.

22. Takita K, Morimoto Y, Okamura A, Kemmotsu O. Do age-based formulae predict the appropriate endotracheal tube sizes in Japanese children? J Anaesth. 2001; 15(3):145-48. DOI: 10.1007/ s005400170016; PMID: 14566512.

23. Finholt DA, Henry DB, Raphaely RC. A "leak" test: a standard method for assessing tracheal tube fit in pediatric patients. Anesthesiology. 1984; 61:450.

24. Covidien. (2011). Tracheostomy Products Quick Reference Guide. [Retrieved 2017 Feb 27]. Available from: : https://www.vitalitymedical.com/pdf/shiley-quick-ref-guide.pdf.

25. Covidien. (2015). Tracheostomy Products Quick Reference Guide. [Retrieved 2017 Feb 27]. Available from: http://www.medtronic.com/content/dam/covidien/library/us/en/ legacyimport/patientmonitoringrecovery/airway $/ 1 /$ shiley-tracheostomy-products-quickreference-guide.pdf. 